\title{
Changes in quality of life among elderly patients with hip fracture in Taiwan
}

\author{
Yea-Ing Lotus Shyu · Min-Chi Chen · Jersey Liang \\ Jui-fen Rachel Lu · Chi-Chuan Wu · Juin-Yih Su
}

Received: 10 March 2003 / Accepted: 24 September 2003/Published online: 7 November 2003

(C) International Osteoporosis Foundation and National Osteoporosis Foundation 2003

\begin{abstract}
To examine the longitudinal change in health related quality of life (HRQoL) during 1 year following hospital discharge in elderly subjects, 110 hip fractured subjects (age, mean \pm SD: $79.3 \pm 7.4$ years) were enrolled in a prospective study. Face-to-face interviews with the patients were conducted, using Short Form 36 (SF-36) at $1,3,6$, and 12 months after they were discharged from the hospital. The GEE approach was employed to evaluate changes in the variables of interest among different time points. Subjects in this study appeared to have lower scores in most dimensions of SF-36, with physical function and role limitation being the lowest due to physical problems (mean $\pm \mathrm{SD}=10.97 \pm 16.19$; $6.32 \pm 20.60)$ during the 1st month after hospital discharge, compared to community dwelling subjects
\end{abstract}

Y.-I.L. Shyu ( $\square)$

Center for Gerontological Research, School of Nursing, Chang Gung University, 259 Wen-Hua 1st Road,

Kwei-Shan, 333 Tao-Yuan, Taiwan, ROC

E-mail: yeaing@mail.cgu.edu.tw

Tel.: + 886-3-2118800 ext. 5275

Fax: $+886-3-2118400$

M.-C. Chen

Department of Public Health, Chang Gung University, 259 Wen-Hua 1st Road, Kwei-Shan,

333 Tao-Yuan, Taiwan, ROC

J. Liang

Health Management and Policy, School of Public Health, M3234 School of Public Health II,

University of Michigan, Ann Arbor, MI 48109-2029, USA

J.R. Lu

Center for Health Industry Management and Public Policy, Department of Health Care Management, Chang Gung University, 259 Wen-Hu 1st Road, Kwei-Shan, 333 Tao-Yuan, Taiwan, ROC

C.-C. $\mathrm{Wu}$

Department of Orthopedics, Chang Gung Memorial Hospital,

5 Fu-Hsin Street, Kwei-Shan, Tao-Yuan, Taiwan, ROC

J.-Y. Su

Department of Trauma and Emergency Surgery, Chang Gung Memorial Hospital, 5 Fu-Shin Street,

Kwei-Shan, Tao-Yuan, Taiwan, ROC (mean $\pm \mathrm{SD}=77.5 \pm 20.5 ; 63.8 \pm 45.30$ ). Most of the dimensions of SF-36, except general health (6th month versus 3 rd month $=57.56 \pm 21.90$ versus $61.75 \pm 23.46$, $P>0.05)$ improved significantly from the 1 st month to the 3rd month (range of means of improved scores from 12.81 to $30.76, P<0.01)$. After the 3 rd month after discharge, physical functions kept improving significantly until 6 months after hospital discharge (3rd month versus 6th month $=25.18 \pm 23.66$ versus $40.30 \pm 25.94, P<0.05)$. Role limitation due to physical problems reached a plateau between the 3rd and 6th month, and then again improved significantly during the 6th month and the 1st year after hospital discharge (6th month versus 1 st year $=17.69 \pm 31.78$ versus $32.22 \pm 44.47, P<0.05)$. The rest of the dimensions of SF-36 remained stable from the 3rd month to 1 year after discharge. These results indicated that different aspects of SF-36 recovered differently for the hip fractured patients in Taiwan. Similar studies may be helpful for health-care providers in other countries with Chinese populations to develop specific intervention programs.

Keywords Elderly persons - Health related quality of life $\cdot$ Hip fracture $\cdot$ Longitudinal change

\section{Introduction}

Hip fracture is a serious consequence of osteoporosis in the elderly because it leads to excess mortality of 5-20\%, and morbidity that results in huge economic costs [1]. The percentage of elderly persons $(1,973,000)$ in Taiwan has increased steadily, from $2.7 \%$ in 1966 to $8.8 \%$ in 2001 [2]. With an increasing aging population, hip fractures represent a major and growing health care problem in Taiwan. According to Tsai et al. [3], it is estimated that the incidence rate (per 100,000) of hip fracture in Taiwan was 211 for the population aged 65 and over (128 in women; 83 in men) in 1993. Tsai and his colleagues reported that despite the use of advanced treatment, the mortality rate remains significant, and 
that a majority of the patients will never recover completely in terms of activities of their daily living functions. The economic cost of hip fractures in the elderly is huge in Taiwan [3], and the families suffer under the extra burden of caregiving.

The self-rated Health-Related Quality of Life (HRQoL) method has been used to supplement traditional indicators such as mortality and objective clinical parameters to assess the effect of illness and the outcome of treatments [4,5]. In addition to the objective indicators evaluated by health care providers, the HRQoL will be able to provide subjective evaluation of health and related life domains from the client's perspective, to enhance our understanding of the impact of the hip fracture or the effect of the treatments to the lives of elderly persons. The HRQoL measurement has been found to be valid and sensitive in predicting mortality in the elderly, and in detecting functional impairment [6]. Some studies found that elderly patients with hip fracture reported lower or poorer HRQoL than those without $[1,7,8]$.

The vast majority of current findings concerning the HRQoL of hip fractured elderly patients are based on data collected in Western developed countries, but little is known about hip fractured elderly patients in Asian countries. It is crucial to evaluate the external validity of these observations with data derived from other societies and cultures, as HRQoL can also be viewed as part of the social and cultural fabric of a people, and may vary significantly across different cultures. For example, vitality, mental health and social functioning might have different presentation in elderly patients in a Chinese society like Taiwan from elderly patients in Western society. Taiwan differs substantially from Western countries in its health care system, clinical practice, casemix, culture, and social organization. For example, the fact Taiwan has national health insurance and the people have the choice of visiting a clinic in a medical center without a referral, might make the needs and health behaviors of elderly patients with a hip fracture different from their counterparts in Western countries. In addition, around $90 \%$ of dependent elderly persons in Taiwan are cared for by family members [9]. Chinese culture emphasizes devotion to parents, social orientation and interdependence, whereas Western culture values autonomy and independence and does not encourage over-sacrifice for parents [10]. These differences might cause the different presentation of quality of life among hip fractured elderly patients in Taiwan compared with those in Western countries. The similarities and differences in the findings from Taiwan and those from the Western countries are useful for further scientific understanding and clinical implementation.

The purpose of this study was to describe the postoperative trend of the HRQoL for a sample of hip fractured elderly patients from a medical center in Taiwan during a period of 12 months following their hospital discharge. By using data from more than two time points with statistical method of longitudinal data analysis, this study is able to fill the gap in previous knowledge and provide information on the changes in HRQoL of elderly hip fractured persons in Taiwan. The analysis was based on data from a prospective study on hip fractured elderly patients admitted to a 3800 bed medical center in Northern Taiwan. We used the Medical Outcomes Study Short Form (SF-36) to measure the HRQoL of hip fractured elderly patients in this study. The changes in HRQoL, including physical and social functioning, mental health, role limitations due to physical and emotional problems, bodily pain, general health perception, and vitality were explored, respectively.

\section{Materials and methods}

A prospective cohort study design was used to explore the longitudinal changes of HRQoL of hip fractured elderly patients. Data were collected from a sample of 110 elderly patients with a hip fracture from a medical center at 1, 3, 6 and 12 months after hospital discharge, by means of face-to-face interviews.

Study setting and sample

This study was conducted in the trauma wards of a 3800-bed medical center in northern Taiwan, as well as in home settings. The inclusion criteria were: 1) aged 65 years or older; 2) hospitalized due to a hip fracture, and having received surgery for internal repairs, or arthroplasty; 3) living geographically in northern Taiwan; and 4) no cognitive impairment by judgement of the treating physician. One hundred and thirty subjects who met the research criteria were invited to participate in the study, but $20(15.38 \%)$ refused. One hundred and ten subjects were enrolled in the study and interviewed before hospital discharge, and at 1, 3, 6 and 12 months after hospital discharge.

\section{Procedures}

Approval from the institution was obtained before collecting the data. Research assistants identified potential subjects after they received surgery and before they were discharged from the hospital, and invited them to participate in this study. Elderly patients who agreed to participate received face-to-face interviews.

Due to the high illiteracy rate $(57.8 \%)$ among the older people in Taiwan [11] face-to-face interviews were used to collect data. Training programs were provided for the interviewers, and regular research team meetings were used to monitor the quality of the data obtained. Two registered nurses were hired as interviewers for this study, and they attended two training sessions. The first session focused on the introduction of the instrument, and a demonstration of how a proper interview was to be conducted. The second training session included an actual interview practice. Any problems raised during the data collection process were discussed and resolved on a regular basis.

Instruments

Pre-fracture self-care ability

Self-care ability before hip fracture was assessed by the performance of pre-fracture activities of daily living (ADL) such as walking, bathing, climbing stairs, transferring, grooming and eating, which were measured using the Chinese Barthel Index (CBI). 
The CBI was translated by Chen and colleagues [12], and is reported as having established reliability and validity, and as being appropriate for assessing frail elderly patients in Taiwan. In this study, Cronbach's alpha of the CBI was 0.88 .

\section{Medical Outcomes Study Short Form (SF-36)}

The SF-36 is a widely used profile measure of generic HRQoL and allows further comparison among patients across different countries and/or with a different disease $[13,14]$. The SF-36 consists of 36 items representing eight generic health concepts: physical functioning (PF), role disability due to physical health problems (RP); bodily pain (BP); vitality (energy/fatigue) (VT); general health perceptions $(\mathrm{GH})$; social functioning $(\mathrm{SF})$; role disability due to emotional problems (RE); and general mental health (MH). For each scale, reverse items were recoded, the simple algebraic sums were then computed, and then the raw scale scores were transformed to a $0-100$ scale. For the final score, the higher the score, the better the implied health related quality of life. In addition, one item of reported health transition (HT) in SF-36 was included as well. This five-level item asked respondents the amount of change in their general health as compared to 1 year ago. The higher the score the more respondents believed that their general health was worse now than 1 year ago. The scores of this item were not transformed. Finally, physical (PCS) and mental (MCS) component summary scales using norm-based $(50,10)$ scoring methods were calculated [15]. In order to examine the relative mental and physical function of the hip fractured elderly patients in comparison with the general population of Taiwan, Taiwan-specific SF-36 algorithms based on a previous study [16] were used to compute the PCS and MCS. The good validity and reliability of SF-36 among the elderly population in the United States have been reported $[13,14,17]$. The SF-36, Taiwan version, was translated and was demonstrated to have good reliability and validity in a sample of total hip replacement patients $[14,18]$. The internal consistency of the eight scales ranged from 0.41 to 0.84 in this study, with role emotion being 0.41 , role physical being 0.56 , and others above 0.62 .

\section{Statistical analysis}

For subjects who answered more than half of the items of the subscale, a person-specific estimate was substituted for any missing item in this subscale. This estimate was suggested by Ware et al. [19], and calculated as the average score across competed items in the same scale. For the elderly patients who died or dropped out within a year following discharge, their data before death and dropping out were included for the analysis. We found no differences between subjects who died within 1 month and those who did not, with regard to pre-fracture self-care ability, hospital stay and demographic variables. The generalized estimating equations (GEE) $[20,21]$ approach was used to determine whether each dimension of HRQoL differed among the 1, 3, 6, and 12 months after hospital discharge, since this method accounts for possible correlations in repeated measures over time and is able to explore the differences among different time points. Its application in our study is to model dependent variables (dimensions of HRQoL) as a function of "time", pre-fracture self-care ability, and hospital discharge. The latter two variables were included in the GEE models as covariates because they were significant in the univariate analysis and were consistent predictors for HRQoL and functional recovery in many literatures as well $[5,22,23]$. The changes in the item of health transition were not analyzed by GEE, due to the small range of the potential scores. All data were analyzed by SAS Win 8.0.

\section{Results}

There were $67(60.9 \%)$ females and $43(39.1 \%)$ males. The age of the subjects ranged from 65 to 96 years, with a mean age of $79.43(\mathrm{SD}=7.51)$ years. Seventy-eight $(70.9 \%)$ had no formal education, $18(16.4 \%)$ completed primary school (1-6 years), $12(10.9 \%)$ completed high school ( $7-13$ years), and two $(1.8 \%)$ had a college $(>13$ years) education. Seventy $(63.6 \%)$ subjects had received internal fixation and $40(36.4 \%)$ received arthroplasty. Average length of hospital stay was $12.40(\mathrm{SD}=5.41)$ days. Average pre-fracture ADL was $96.09(\mathrm{SD}=11.48)$, representing relatively independent pre-fracture physical functioning. Eighteen subjects $(16.4 \%)$ died during 1 year following discharge that eight subjects $(7.3 \%)$ died within the 1 st month, five subjects $(4.5 \%)$ died between the 1st and 3rd months, two subjects $(1.8 \%)$ died between the 3rd and 6th months, and three subjects $(2.7 \%)$ died between 6th and 12th months. In addition, one subject $(0.9 \%)$ at the 1 st month, four subjects $(3.6 \%)$ at the 3 rd month, six subjects $(5.5 \%)$ at the 6th month and 14 subjects $(12.7 \%)$ at the 12 th month dropped out from the study due to refusal or lost contact. Five $(4.5 \%)$ of the subjects were institutionalized before the hip fracture, 11 subjects $(10.0 \%)$ were institutionalized during the 1 st month after discharge, ten $(9.1 \%)$ between the 1 st and 3rd months, and four (3.6\%) between the 3rd and 6th months, and also between the 6th and 12th months after discharge. In terms of performance in SF-36 from the 1st to 12th month after discharge, the mean improved from 58.4 to 75.2 for BP, from 57.6 to 55.1 for $\mathrm{GH}$, from 45.2 to 55.1 for VT, from 52.2 to 65.3 for SF, from 46.7 to 90 for RE, from 53.8 to 72.3 for $\mathrm{MH}$, from 11.0 to 40.8 for PF and from 6.3 to 32.2 for RP (Table 1). We found no differences in almost all HRQoL subscales at the 1st, 3rd, and 6th months after discharge between subjects who remained in the study throughout the 12 months and those who dropped out later between the 6th month and 12th month.

\section{Comparison with the norm}

To show as a whole, the degree of recovery of hip fracture patients of eight subscales except health transition during a 1-year period, transformed to a 1-100 scale, were compared with a norm of community dwelling Taiwanese elderly patients $(n=1559)$ who were aged 65 or older (Fig. 1). In comparison with the norm, the hip fractured elderly patients seemed to have considerably poorer performance regarding bodily pain, vitality, social function, role emotion, mental health, physical function and role physical at the 1st month after being discharged from the hospital. Physical function and role limitations due to physical problems (role physical) of hip fractured elderly patients (mean $\pm \mathrm{SD}=10.97 \pm 16.19 ; 6.32 \pm 20.60$ ) were the two subscales that deviated the most from the norm (mean $\pm \mathrm{SD}=77.5 \pm 20.5 ; 63.8 \pm 45.3$ ) among the elderly patients living within the community. From the 4 th month onwards up to 1 year after their hospital discharge, the performance of the hip fractured elderly patients improved to the same level as their community 
Table 1 Means, standard deviations, and related variables of each SF36 subscale at each time point during 1 year after discharge. $P$-value was obtained using the GEE approach and denotes the significance between three time points and baseline and the regression coefficient

${ }^{\text {a}}$ Estimate of regression coefficient from GEE approach

\begin{tabular}{|c|c|c|c|c|c|c|}
\hline \multirow{2}{*}{$\begin{array}{l}\text { Variables } \\
\text { SF36 } \\
\text { subscale }\end{array}$} & \multicolumn{4}{|c|}{ Time $($ mean $\pm S D)$} & \multirow{2}{*}{$\begin{array}{l}\text { Hospital } \\
\text { stay }^{\mathrm{a}}\end{array}$} & \multirow{2}{*}{$\begin{array}{l}\text { Pre-fracture } \\
\text { ADL* }\end{array}$} \\
\hline & Month 1 & Month 3 & Month 6 & 1 year & & \\
\hline BP & $\begin{array}{l}58.4 \pm 22.45 \\
\text { (Baseline) }\end{array}$ & $\begin{array}{l}70.0 \pm 22.35 \\
(P<0.0001)\end{array}$ & $\begin{array}{l}78.4 \pm 23.67 \\
(P<0.0001)\end{array}$ & $\begin{array}{l}75.2 \pm 25.48 \\
(P<0.0001)\end{array}$ & $\begin{array}{l}-1.4549 \\
(P<0.0001)\end{array}$ & $\begin{array}{l}0.2501 \\
(p=0.3368)\end{array}$ \\
\hline GH & $\begin{array}{l}57.56 \pm 21.90 \\
\text { (Baseline) }\end{array}$ & $\begin{array}{l}61.75 \pm 23.46 \\
(P=0.2415)\end{array}$ & $\begin{array}{l}60.32 \pm 21.62 \\
(P=0.7004)\end{array}$ & $\begin{array}{l}55.08 \pm 20.87 \\
(P=0.4104)\end{array}$ & $\begin{array}{l}-0.6549 \\
(P=0.1051)\end{array}$ & $\begin{array}{l}0.7709 \\
(p=0.0043)\end{array}$ \\
\hline VT & $\begin{array}{l}45.17 \pm 22.83 \\
\text { (Baseline) }\end{array}$ & $\begin{array}{l}59.78 \pm 21.83 \\
(P<0.0001)\end{array}$ & $\begin{array}{l}61.04 \pm 21.97 \\
(P=0.0006)\end{array}$ & $\begin{array}{l}55.11 \pm 20.77 \\
(P=0.0235)\end{array}$ & $\begin{array}{l}-0.3645 \\
(P=-0.3219)\end{array}$ & $\begin{array}{l}0.6067 \\
(p=0.0759)\end{array}$ \\
\hline SF & $\begin{array}{l}52.15 \pm 27.57 \\
\text { (Baseline) }\end{array}$ & $\begin{array}{l}64.37 \pm 24.84 \\
(P=0.0001)\end{array}$ & $\begin{array}{l}65.15 \pm 29.13 \\
(P=0.0248)\end{array}$ & $\begin{array}{l}65.27 \pm 27.68 \\
(P=0.0196)\end{array}$ & $\begin{array}{l}-1.0215 \\
(P=0.0144)\end{array}$ & $\begin{array}{l}0.3670 \\
(p=0.1929)\end{array}$ \\
\hline $\mathrm{RE}$ & $\begin{array}{l}46.74 \pm 48.61 \\
\text { (Baseline) }\end{array}$ & $\begin{array}{l}75.52 \pm 41.92 \\
(P<0.0001)\end{array}$ & $\begin{array}{l}83.58 \pm 34.42 \\
(P<0.0001)\end{array}$ & $\begin{array}{l}90.00 \pm 30.38 \\
(P<0.0001)\end{array}$ & $\begin{array}{l}-2.2879 \\
(P<0.0001)\end{array}$ & $\begin{array}{l}0.4516 \\
(\mathrm{p}=0.3461)\end{array}$ \\
\hline MH & $\begin{array}{l}53.82 \pm 19.56 \\
\text { (Baseline) }\end{array}$ & $\begin{array}{l}66.98 \pm 19.42 \\
(P<0.0001)\end{array}$ & $\begin{array}{l}69.50 \pm 17.98 \\
(P<0.0001)\end{array}$ & $\begin{array}{l}72.78 \pm 18.12 \\
(P<0.0001)\end{array}$ & $\begin{array}{l}-0.9516 \\
(P=0.0064)\end{array}$ & $\begin{array}{l}0.4284 \\
(\mathrm{p}=0.1128)\end{array}$ \\
\hline PF & $\begin{array}{l}10.97 \pm 16.19 \\
\text { (Baseline) }\end{array}$ & $\begin{array}{l}25.18 \pm 23.66 \\
(P<0.0001)\end{array}$ & $\begin{array}{l}40.30 \pm 25.94 \\
(P<0.0001)\end{array}$ & $\begin{array}{l}40.76 \pm 29.92 \\
(P<0.0001)\end{array}$ & $\begin{array}{l}-1.0698 \\
(P=0.0008)\end{array}$ & $\begin{array}{l}0.7604 \\
(p<0.0001)\end{array}$ \\
\hline $\mathrm{RP}$ & $\begin{array}{l}6.32 \pm 20.60 \\
\text { (Baseline) }\end{array}$ & $\begin{array}{l}18.98 \pm 34.94 \\
(P=0.0260)\end{array}$ & $\begin{array}{l}17.69 \pm 31.78 \\
(P=0.0653)\end{array}$ & $\begin{array}{l}32.22 \pm 44.47 \\
(P=0.0034)\end{array}$ & $\begin{array}{l}-1.4141 \\
(P=0.0031)\end{array}$ & $\begin{array}{l}-0.2199 \\
(p=0.5803)\end{array}$ \\
\hline
\end{tabular}

Fig. 1 Comparison of HRQoL of hip fractured elderly patients within the norm [41]. BP bodily pain; $G H$ general health; $V T$ vitality; $S F$ social functioning; $R E$ role emotional; $M H$ mental health; $P F$ physical functioning; $R P$ role physical

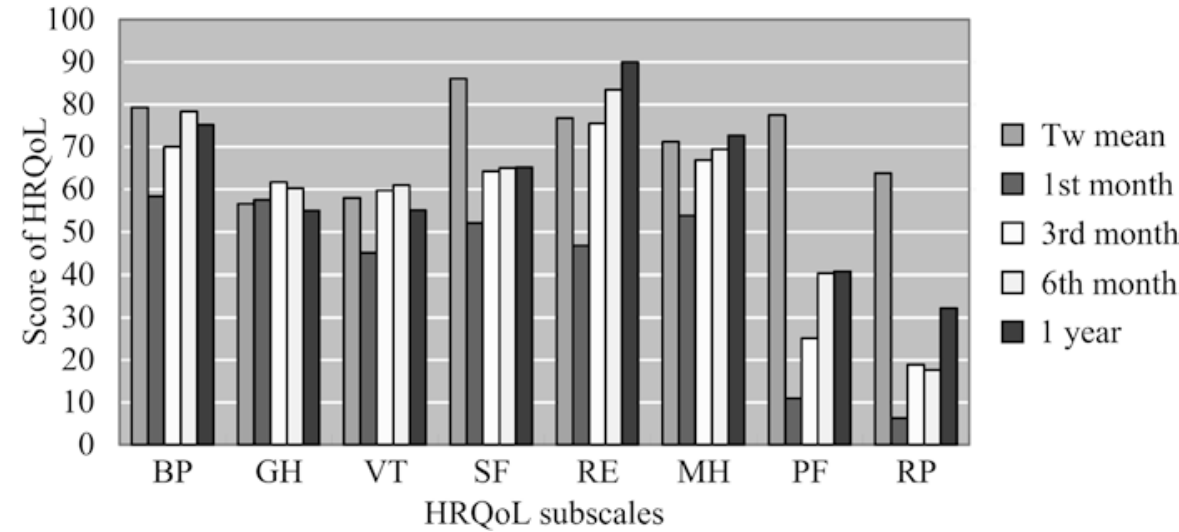

dwelling counterparts, in most of the subscales except social function, physical function and role physical. The gap between hip fractured elderly patients and community dwelling elderly patients at social function got closer from the 1st to the 3rd month, then stayed the same until 1 year following discharge. The gap in physical function between hip fractured elderly patients and the norm became closer from the 1st to 6th month and stayed the same until 1 year following hospital discharge. The gap of role physical between hip fractured elderly patients and the norm got closer from the 1st to 3rd month, and from 6 months to 1 year following discharge.

In examining the relative mental and physical function of the hip fractured elderly patients in this sample in comparison with the general population of Taiwan, norm-based scores of PCS and MCS were calculated by using parameters derived from a general Taiwan population [16]. The mean of PCS was $15.60(\mathrm{SD}=8.82)$ at the 1st month, $22.42(\mathrm{SD}=11.61)$ at the 3rd month, $25.39(\mathrm{SD}=12.34)$ at the 6th month, and 28.04 $(\mathrm{SD}=13.20)$ at 1 year after discharge. For the mean of the MCS, $49.83(\mathrm{SD}=12.54)$ at the 1st month, 55.04 $(\mathrm{SD}=11.41)$ at the 3rd month, $54.91(\mathrm{SD}=9.55)$ at the 6th month, and $54.35(\mathrm{SD}=9.75)$ at 1 year after discharge were found.

\section{Longitudinal changes}

Longitudinal changes of eight subscales, except health transition transformed to a 1-100 scale at the 1st, 3rd, 6th and 12th months after hospital discharge, are presented in the graph in Fig. 2. Physical related subscales, except bodily pain such as physical functioning and role physical, had the poorest performance among all the subscales. On the other hand, mental/social related subscales such as role emotion, mental health, and social function appeared to have a better performance. At the same time, general health and vitality, which might be influenced by both mental and physical conditions, performed better than the physical related subscales, but poorer than the mental related subscales. However, bodily pain that is also physically related appeared to be as good as the mentally related dimensions for these hip fractured elderly patients within 1 year after hospital discharge. 
Fig. 2 Longitudinal changes of HRQoL during 1 year after hospital discharge. $B P$ bodily pain; $G H$ general health; $V T$ vitality; $S F$ social functioning; $R E$ role emotional; $M H$ mental health; $P F$ physical functioning; $R P$ role physical

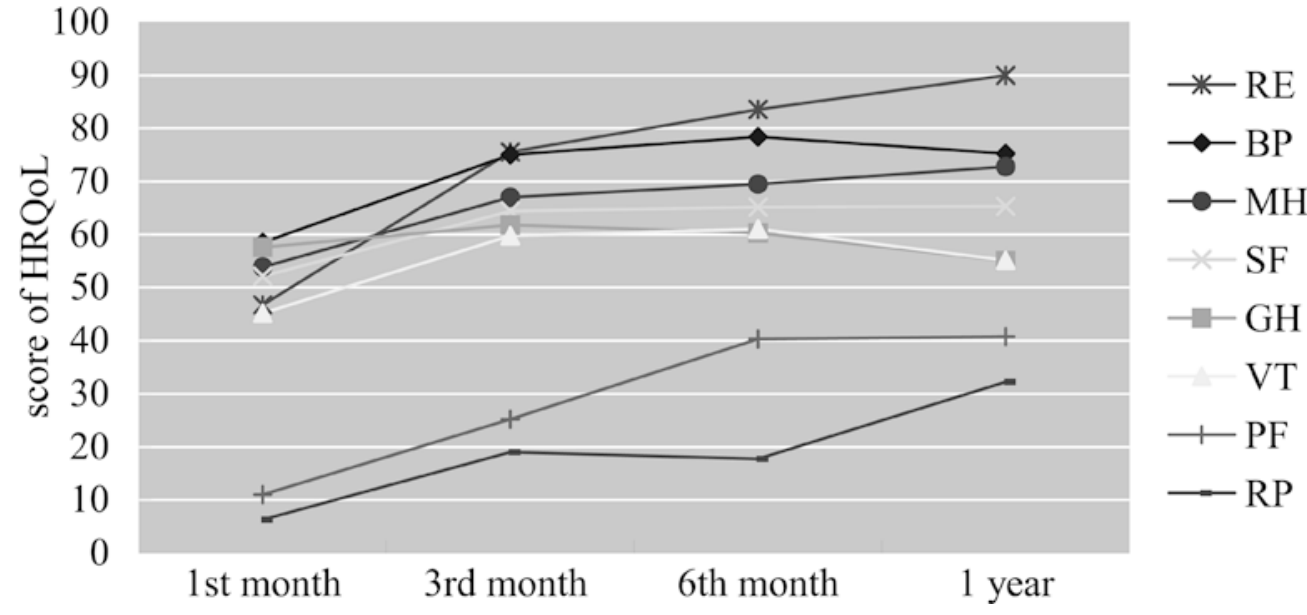

The means of each subscale at each time point are listed in Table 1. The significance of the differences of the trends was examined by using GEE. Only the general health subscale did not have any significant changes throughout the follow-up period from the 1st month to 1 year after being discharged from the hospital. On the other hand, when compared to 1 year ago (health transition), the subjects reported their general health from "somewhat worse" during the 1st month after their discharge (mean $\pm \mathrm{SD}=3.81 \pm 0.93$ ) to partway between "somewhat worse" and "about the same" after the 3rd month (mean $\pm \mathrm{SD}=3.58 \pm 0.81$ ); it then improved to "about the same" at the 6th month (mean $\pm \mathrm{SD}=3.15 \pm 0.99$ ), and then improved little from the 6th month to 1 year (mean $\pm \mathrm{SD}=2.97 \pm 1.13$ ) after discharge.

Most of the remaining subscales, including bodily pain, vitality, social function, role emotion, and mental health, improved significantly from the 1st month to the 3rd month and then remained stable for the rest of the year. So, when using the 1st month after their discharge as the baseline, the scores of these subscales at the other time points were significantly higher (range of means of improved scores from 12.81 to $30.76, P<0.01$ ). However, when using the 3rd month after discharge as the baseline, the scores at either the 6th month or at 1 year after discharge did not differ significantly. When using the 6th month after discharge as the baseline, the scores of these subscales at 1 year after discharge did not differ significantly either. Different change patterns were observed in physical function and role physical subscales where the scores of these two subscales increased significantly not only from the 1st to 3rd month, but also afterwards. After the 3rd month after discharge, physical function kept improving significantly until 6 months after hospital discharge (3rd month versus 6th month $=25.18 \pm 23.66$ versus $40.30 \pm 25.94, P<0.0001)$. After 6 months, the physical function of the hip fractured elderly patients remained stable until 1 year after their discharge from the hospital. For the role physical subscale, in addition to the improvement from the 1st to the 3rd month, a significant improvement was also found from the 6th month to 1 year (6th month versus 12 th month $=17.69 \pm 31.78$ versus $32.22 \pm 44.47$, $P<0.05)$. The role limitation due to physical function for the hip fractured elderly patients from the 3 rd to the 6th month did not change significantly.

\section{Related factors}

The trends in the subscales of SF-36 were first interpreted after controlling for hospital stay and pre-fracture ADLs. It appeared that most of the subscales, except vitality, general health and health transition, were related to the length of hospital stay. The longer the hospital stay, the more these patients experienced interference with normal activity due to pain, increasing role limitation due to emotional and physical problems, and a worsening of their mental health, social and physical functions over a 1-year period following discharge. At the same time, hip fractured elderly patients who had worse pre-fracture ADLs appeared to have poorer general health and physical function and also poorer perception of health as compared to 1 year ago during the 1-year period following discharge. Vitality was not related to either the length of hospital stay or pre-fracture ADLs.

We further added age and gender in addition to hospital stay and pre-fracture ADL into the GEE model. After controlling for hospital stay, pre-fracture ADLs and gender, age did not predict the trends of any dimension of HRQoL $(P=0.74$ for BP; 0.28 for $\mathrm{GH}$; 0.37 for VT; 0.55 for SF; 0.97 for RE; 0.68 for $\mathrm{MH} ; 0.79$ for PF; 0.10 for RP). After controlling for hospital stay, pre-fracture ADL, and age, male appeared to have less interference with normal activity due to pain $(P=0.044)$, and better physical function $(P=0.47)$. Males and females were not significantly different in trend of general health $(P=0.14)$, vitality $(P=0.40)$, social function $(P=0.08)$; role emotion $(P=0.13)$; mental health $(P=0.06)$, and role physical $(P=0.16)$. 


\section{Discussion}

Mortality has been examined in many studies as one of the important possible postoperative outcomes for elderly persons with a hip fracture $[24,25,26,27,28,29,30]$. However, although the mortality rate is an important indicator for health statistics, it is not sensitive to changes in treatment or post-surgery interventions, and can only provide very limited information on the conditions of the hip fractured elderly patients. Functional recovery and clinical complications after the operation of elderly persons with hip fracture have been examined extensively [22,23,31,32,33,34]. However, functional recovery and clinical complications are not able to provide information from the perspective of the elderly persons themselves. The HRQoL is able to provide subjective evaluation of health and related life domains from the perspective of the elderly persons to supplement the functional and clinical indicators, and can provide a holistic view of the effect of hip fracture and the outcome of interventions.

Hip fracture, one of the most severe consequences of osteoporosis, has been reported as being associated with a deterioration in all dimensions of HRQoL, including general health, psychological well-being, physical and social functioning [1]. However, these consequences have not yet been established in Chinese elderly patients with hip fracture. As in studies from Western countries $[1,7,8,33]$, this study suggests that Chinese elderly patients with hip fracture experience a significant deterioration in most dimensions of HRQoL, especially during the 1st month after being discharge from hospital. In comparison with the norm of the community dwelling elder in Taiwan, results of this study indicated that most dimensions of HRQoL for Chinese hip fractured elderly patients were relatively low after 1 month, with physical related functioning being the lowest. These elderly patients also reported their general health being somewhat worse than 1 year ago at 1 month after their hospital discharge. As found in previous studies $[3,22]$ the physical function, role limitation due to physical problems, and the social function of hip fractured elderly patients were all poorer than that of elderly patients living within the community, even 1 year after being discharged from the hospital. In comparison with general Taiwanese population, hip fractured elderly patients in this sample had very low overall physical function as demonstrated by PCS through the 1st year, which indicated substantial limitations in self-care, physical, social and role activities, frequent tiredness, more bodily pain and health in general rated poor [15]. The mental function of the hip fractured elderly patients as demonstrated by MCS throughout the 1st year after discharge was similar to the general population of Taiwan.

Significant improvement during the first 3 months after hospital discharge for most dimensions of HRQoL was supported by a previous study, which found that after hospital admission, there was an improvement of quality of life in the follow-up period of 4 months [35]. Three months after their hospital discharge, we found that the patients' physical function kept improving significantly till the 6th month. Also, hip fractured elderly patients did not report their general health being the same as 1 year ago until 6 months after their hospital discharge. A previous study reports that most of the recovery in walking ability and ability to perform ADL occurs within the first 6 months [22]. This study also found that most dimensions of HRQoL did not change significantly from the period of 6 months to 1 year after discharge, and this was supported by a previous study [36]. Differing from this previous study, we found that role limitation due to physical problems improved during the first 3 months, attained a plateau between the 3rd and the 6th month, and then improved significantly again between 6 months and 1 year after hospital discharge. Whether this difference is due to cultural factors will need to be explored further. For example, regardless of rapid economic development and social change in Taiwan since the end of World War II, mutual support kinship relationship and filial piety of respecting and supporting elderly parents are still highly valued among modern Taiwanese [37,38]. In the sample of this study, only $10 \%$ of the hip fractured elderly patients were institutionalized during the 1st month following hospital discharge, and this percentage decreased to $9.1 \%$ at the $3 \mathrm{rd}$ month, and $3.6 \%$ at the 6 th month after discharge; only one $(0.9 \%)$ patient lived alone during this period. The majority of elderly patients in Taiwan continue to reside with their adult, married children [39]. It would be interesting to study whether the family condition and living arrangement influences the performance of independent role functions of elderly persons, even when they are physically capable.

The fact that the pre-fracture ADL influenced the physical function can be supported by a previous study in which pre-fracture ADL had a significant association with ADL functional recovery over the course of 1 year [23]. The association between hospital stay and functional recovery after hip fracture has also been reported previously $[22,23,40]$. Also being supported by a previous study [23] is the fact that the type of surgical procedure was dropped from the model in this study because of its insignificant value to this study.

These results indicated that different aspects of HRQoL recovered differently for elderly patients who suffered a hip fracture. The physical function that appeared to make the greatest impact was the ability to keep improving for 6 months after the discharge. In addition, improvement of role limitation due to physical problems occurred later than the improvement in physical function. Based on these findings, several suggestions can be made for clinical practice in taking care of Chinese hip fractured elderly patients. First of all, a hip fracture had an impact on not only patients' physical aspects, but on all aspects of HRQoL for these elderly patients. Health care professionals need to pay attention 
to all these aspects of HRQoL. Secondly, post-fracture intervention programs should be developed and evaluated as to whether they are useful in improving physical function and other $\mathrm{HQoL}$ aspects, and intervention studies should probably focus on early intervention. In Taiwan, hip fractured patients usually discharge from the hospital around 7 days after the surgery. After the hospital discharge, no in-home programs for rehabilitation or nursing care are provided in routine care. Patients usually come back to the clinic around 1 month, 3 months, 6 months and 1 year after the hospital discharge for follow-ups and treatment for osteoporosis if needed. However, adherence to the follow-up schedule is poor. Finally, interventions can and should be developed to facilitate the hip fractured elderly patients in developing effective coping strategies, as well as a realistic perception about their physical problems, in order to speed up improvement of their role functioning, which is affected by their physical limitations.

In summary, in a Taiwanese sample, the longitudinal changes of HRQoL of hip fractured elderly patients were explored. This study was limited by lack of data collection on the pre-fracture HRQoL, the use of a convenience sample, and the lack of a true control population of elderly patients. The similarity between the profile of this sample and the sample from Tsai and colleagues' study of hip fractured patients in Taiwan [3] might lessen the impact of a convenience sample on the generalizability of the results. For example, the proportion of male subjects $(39.1 \%)$ in this study is comparable with the incidence rate for elderly men among the total elderly population (39.4\%) in 1993 as reported by Tsai and colleagues [3]. Future studies with a random sample and with collection of a pre-fracture HRQoL can be further developed to explore this phenomenon. Interventional studies based on the findings of this study could also be conducted afterwards. Similar studies may be helpful for health-care providers in other countries with Chinese populations, to develop specific intervention programs.

Acknowledgements This work was funded by the National Health Research Institute, Republic of China.

\section{References}

1. Randell AG, Nguyen TV, Bhalerao N et al (2000) Deterioration in quality of life following hip fracture: a prospective study. Osteoporos Int 11:460-466

2. Directorate-General of Budget, Accounting and Statistics, Executive Yuan \& Ministry of Interior, ROC (nd) (2001) Social indicators contents: age-specific distribution of population. Retrieved February 14, 2003, from http://www.dgbas.gov.tw/ dgbas03/bs2/91chy/table/s003.xls

3. Tsai YJ, Lin HS, Chow LP (1995) A pilot study on health sector priority review in Taiwan: by retrospective study on the elderly hip fracture (monograph). Taiwan Provincial Institute of Family Planning, Taichung, Taiwan
4. Greenfield S, Nelson EC (1992) Recent developments and future issues in the use of health status assessment measures in clinical settings. Med Care 30:MS23-41

5. Wilson IB, Cleary PD (1995) Linking clinical variables with health related quality of life. JAMA 273:59-65

6. Idler EL, Kasl SV, Lemke JH (1990) Self-evaluated health and mortality among the elderly in New Haven, Connecticut, and Iowa and Washington counties, Iowa, 1982-1986. Am J Epidemiol 131:91-103

7. Hall SE, Williams JA, Senior JA et al (2000) Hip fracture outcomes: quality of life and functional status in older adults living in the community. Aust N Z J Med 30:327-332

8. Tosteson ANA, Gabriel SE, Grove MR et al (2000) Impact of hip and vertebral fractures on quality-adjusted life years. Osteoporos Int 12:1042-1049

9. Directorate-General of Budget, Accounting and Statistics, Executive Yuan \& Ministry of Interior, ROC (1997) Report on the old status survey: Taiwan area, Republic of China. Directorate-General of Budget, Accounting and Statistics, Executive Yuan \& Ministry of Interior, Taipei, Taiwan

10. Dai YT, Dimond MF (1998) A cross-cultural comparison and its implications for the well-being of older parents. J Gerontol Nurs 24:13-18

11. Directorate-General of Budget, Accounting and Statistics, Executive Yuan \& Ministry of Interior, ROC (1994) Report on the old status survey: Taiwan area, Republic of China. Directorate-General of Budget, Accounting and Statistics, Taipei, Taiwan

12. Chen YJ, Dai YT, Yang CT et al. (1995) A review and proposal on patient classification in long-term care system. Department of Health, Republic of China, Taipei, Taiwan

13. Weinberger M, Samsa GP, Hanlon JT et al. (1991) An evaluation of a brief health status measure in elderly veterans. J Am Geriatr Soc 39:691-694

14. Lyons RA, Perry HM, Littlepage BN (1994) Evidence for the validity of the Short-form 36 Questionnaire (SF-36) in an elderly population. Age Ageing 23:182-184

15. Ware JE, Kosinski MK, Keller SD (1994) SF-36 physical and mental health summary scales: a user's manual. The Health Institute, New England Medical Center, Boston

16. Tseng HM, Lu JFR, Tsai YJ (2003) Assessment of health-related quality of life (II): norming and validation of SF-36 Taiwan version. Chin J Public Health (in press)

17. McHorney CA (1996) Measuring and monitoring general health status in elderly persons: practical and methodological issues in using the SF-36 Health Survey. Gerontologist 36:571583

18. Chiu HC, Mau LW, Hsu YC et al. (2001) Postoperative 6month and 1-year evaluation of health-related quality of life in total hip replacement patients. J Formos Med Assoc 100:461-5

19. Ware JE, Davies-Avery A, Brook RH (1980) Conceptualization and measurement of health for adults in the Health Insurance Study. Volume VI: analysis of relationships among health status measures. The RAND Corporation, Santa Monica, Calif.

20. Liang KY, Zeger SL (1986) Longitudinal data analysis using generalized linear models. Biometrika 73:13-22

21. Liang KY, Zeger SL (1993) Regression analysis for correlated data. Annu Rev Public Health 14:43-68

22. Magaziner J, Simonsick EM, Kashner TM, et al (1990) Predictors of functional recovery one year follow hospital discharge for hip fracture: a prospective study. J Gerontol 45:M101-107

23. Young Y, Brant L, German P et al (1997) A longitudinal examination of functional recovery among older people with subcapital hip fractures. J Am Geriatr Soc 45:288-294

24. Aharonoff GB, Koval KJ, Skovron ML et al (1997) Hip fractures in the elderly: predictors of one year mortality. J Orthop Trauma 11:162-165

25. Boereboom FT, Raymakers JA, Duursma SA (1992) Mortality and causes of death after hip fractures in The Netherlands. Neth J Med 41:4-10 
26. Jacobsen SJ, Goldberg J, Miles TP et al. (1992) Race and sex differences in mortality following fracture of the hip. Am J Public Health 82:1147-1150

27. Nettleman MD, Alsip J, Schrader M et al. (1996) Predictors of mortality after acute hip fracture. J Gen Int Med 11:765-767

28. Pitto RP (1994) The mortality and social prognosis for hip fractures. A prospective multifactorial study. Int Orthop 18:109-113

29. Schroder HM, Erlandsen M (1993) Age and sex as determinants of mortality after hip fracture: 3895 patients followed for 2.5-18.5 years. J Orthop Trauma 7:525-531

30. Kuokkanen HO, Korkala OL (1992) Factors affecting survival of patients with hip fractures. Acta Orthop Belg 58:425-428

31. Baudoin C, Fardellone P, Bean K et al (1996) Clinical outcomes and mortality after hip fracture: a 2-year follow-up study. Bone 18:S149-157

32. Norton R, Butler M, Robinson E et al. (2000) Declines in physical functioning attributable to hip fracture among older people: a follow-up study of case-control participants. Disabil Rehabil 22:345-351

33. Willig R, Keinänen-Kjukaaniemi S, Jalovaara P (2001) Mortality and quality of life after trochanteric hip fracture. Public Health 115:323-327

34. Williams MA, Oberst MT, Bjorklund BC (1994) Early outcomes after hip fracture among women discharged home and to nursing homes. Res Nurs Health 17:175-183
35. van Balen R, Steyerberg EW, Polder JJ et al. (2001) Hip fracture in elderly patients: outcomes for function, quality of life, and type of residence. Clin Orthop:232-243

36. Borgquist L, Nilsson LT, Lindelow G et al. (1992) Perceived health in hip-fracture patients: a prospective follow-up of 100 patients. Age Ageing 21:109-116

37. Tsai YM, Yi CC (1997) Persistence and changes in Chinese family values: the Taiwanese case. In: Chang LY, Lu YH, Wang FC (eds) (1997) Taiwanese society in 1990s: Taiwan social change survey symposium series II (part II) (The Preparatory Office of the Institute of Sociology, Academia Sinica monograph series: no 1). Institute of Sociology, Academia Sinica, Taipei, Taiwan, pp 123-169

38. Yeh KH (1997) Changes in the Taiwan people's concept of filial piety. In: Chang LY, Lu YH, Wang FC (eds) (1997) Taiwanese society in 1990s: Taiwan social change survey symposium series II (part II) (The Preparatory Office of the Institute of Sociology, Academia Sinica monograph series: no 1). Institute of Sociology, Academia Sinica, Taipei, Taiwan, pp 171-214

39. Lee YJ, Parish WL, Wills R (1994) Sons, daughters, and intergenerational support in Taiwan. Am J Sociol 99:1010-1041

40. Barnes B, Dunovan K (1987) Functional outcomes after hip fracture. Phys Ther 67:1675-1679

41. Wu SC (1997) Investigation of health and long-term care conditions of elderly persons in Taiwan. Department of Health, Taiwan, Republic of China, Taipei, Taiwan 